Theoretical and Mathematical Physics, 163(2): 677-695 (2010)

\title{
LANGEVIN EQUATION OF A FLUID PARTICLE IN WALL-INDUCED TURBULENCE
}

\author{
(C) J. J. H. Brouwers*
}

\begin{abstract}
We derive the Langevin equation describing the stochastic process of fluid particle motion in wall-induced turbulence (turbulent flow in pipes, channels, and boundary layers including the atmospheric surface layer). The analysis is based on the asymptotic behavior at a large Reynolds number. We use the Lagrangian Kolmogorov theory, recently derived asymptotic expressions for the spatial distribution of turbulent energy dissipation, and also newly derived reciprocity relations analogous to the Onsager relations supplemented with recent measurement results. The long-time limit of the derived Langevin equation yields the diffusion equation for admixture dispersion in wall-induced turbulence.
\end{abstract}

Keywords: turbulence, dispersion, Langevin equation, Onsager reciprocity

\section{Introduction}

Fluid flow bounded by walls such as the earth's surface or a pipe wall is often turbulent. Fluid velocities and pressures vary randomly with time and space. Despite many efforts over the past century, there is no general mathematical description of the stochastic process of turbulence. The best that has been achieved is asymptotic descriptions of certain statistical parameters in the limit of large values of the Reynolds number $\operatorname{Re}=u_{*} L \nu^{-1}$, where $u_{*}$ is the shear velocity $\left(u_{*}=\sqrt{\tau_{0} / \rho}\right.$, where $\tau_{0}$ is the mean shear stress exerted on the wall by the fluid and $\rho$ is the fluid density), $\nu$ is the fluid kinematic viscosity, and $L$ is the external length (pipe radius, channel half-width, turbulent boundary layer thickness along a wall or along the earth's surface).

Several asymptotic expressions applicable at Re $\gg 1$ have so far been derived, for example, Kolmogorov's renowned descriptions of the small scales of turbulence [1] and the logarithm law for the mean flow along a wall [2]. But the classical problem of calculating turbulent dispersion remains unsolved. A proper theory exists only for the theoretical abstraction of homogeneous isotropic turbulence. It dates back to [3] written in 1921. But turbulence is an inhomogeneous anisotropic process. In all important cases of turbulent flow, the fixed-point statistical means (i.e., Eulerian averages) of fluid velocities vary considerably in space and direction.

A theory convincingly treating admixture dispersion in inhomogeneous anisotropic turbulence has not yet appeared. The standard approach is to use the Boussinesq hypothesis, which leads to a semiempirical equation for turbulent dispersion [2]. The expressions for the diffusion coefficients in this equation are approximate; they are based on a Reynolds analogy and are fitted to measurement results.

A more fundamental approach starts from a Langevin equation for the fluid particle velocity; this approach is analogous to the description of the Rayleigh particle in molecular dynamics [4]. An important advantage of this method is that it is consistent with the asymptotic structure of turbulent flow at a large Reynolds number. The equation can be made to match the inertial subrange limit of the Lagrangian version

*Eindhoven University of Technology, Eindhoven, Netherlands, e-mail: J.J.H.Brouwers@tue.nl.

Prepared from an English manuscript submitted by the author; for the Russian version, see Teoreticheskaya $i$ Matematicheskaya Fizika, Vol. 163, No. 2, pp. 328-352, May, 2010. Original article submitted December 8, 2009. 
of Kolmogorov's similarity theory [5]. But the big problem concerns the form of the damping function in the Langevin equation. While the damping function is well established for homogeneous isotropic turbulence, a unique expression applicable to anisotropic inhomogeneous turbulence is lacking.

Here, we consider admixture dispersion in inhomogeneous anisotropic wall-induced turbulence. We use the Langevin equation and derive an almost asymptotically exact description of the damping function. We use the Lagrangian Kolmogorov theory, recently derived asymptotic expressions for the spatial distribution of turbulent energy dissipation, and newly derived reciprocity relations analogous to the Onsager symmetry relations. The long-time limit applied to the derived Langevin equation yields an almost exact version of the diffusion equation for admixture dispersion in wall-induced turbulence.

\section{Langevin equation}

Describing the stochastic process of turbulent motion of a fluid particle with the Langevin equation assumes that the fluid particle acceleration behaves as a $\delta$-correlated process. The justification for applying this assumption to turbulence is that accelerations are governed by the small viscous scales, which obey Kolmogorov scaling [5]; this feature is apparently confirmed by experiment [6]. The correlation times of particle acceleration are related to those of velocity as $\mathrm{Re}^{-1 / 2}$. As Re increases, the stochastic process describing the velocity in large-scale turbulence is likely to resemble a Markov process more and more, and it can be modeled by a Langevin equation. The white-noise term in the equation can be described in accordance with the inertial subrange limit of the Kolmogorov nonintermittent Lagrangian similarity theory [5]. The Langevin equation relevant to stochastic turbulence then becomes [7]

$$
\frac{d v_{i}^{\prime}}{d t}=a_{i}^{\prime}\left(\boldsymbol{v}^{\prime}, \boldsymbol{x}\right)+\sqrt{C_{0} \epsilon(\boldsymbol{x})} w_{i}(t), \quad i=1,2,3,
$$

where $\boldsymbol{v}^{\prime}=\boldsymbol{v}^{\prime}(t)$ is the statistical fluctuation of the fluid particle velocity with respect to the Eulerian mean velocity $\boldsymbol{u}^{0}(\boldsymbol{x})$ evaluated at the particle position $\boldsymbol{x}=\boldsymbol{x}(t)$. The velocity is related to the position by

$$
\frac{d x_{i}}{d t}=u_{i}^{0}(\boldsymbol{x})+v_{i}^{\prime}
$$

In (1), $a_{i}^{\prime}=a_{i}^{\prime}\left(\boldsymbol{v}^{\prime}, \boldsymbol{x}\right)$ is the damping function, $C_{0}$ is the universal Kolmogorov constant, $\epsilon=\epsilon(\boldsymbol{x})$ is the energy dissipation rate averaged at a fixed position, and $w_{i}(t)$ is Gaussian white noise of unit intensity. Equations (1) and (2) can be solved for various realizations of $w_{i}(t)$ with appropriate conditions at $t=0$. The statistics of the velocity and position of a tagged fluid particle or passive fluid admixture can then be evaluated by averaging the resulting time records at fixed instants. The means thus obtained are denoted by an overbar. An alternative method for obtaining the same statistical information is to solve the FokkerPlanck equation for the joint probability of the particle velocity and position associated with Eqs. (1) and (2).

Describing particle velocity statistics with the Langevin equation relies on applying ordinary nonintermittent Kolmogorov (K-41) theory. The intermittency effects are not taken into account in such an approach. We could adopt a fractal model based on Kolmogorov's refined similarity theory [8], but the statistical means of the particle displacement, which determine the turbulent dispersion, change little in this case [9], [10]. The intermittency effect is apparent at small (viscous) scales, not at the large (energetic) scales governing the stochastic process describing the velocity in turbulent flows at $\operatorname{Re} \gg 1$ [11]. For these reasons among others, a Langevin model based on K-41 theory is considered a reasonable approach for modeling dispersion. 
To predict the statistics of particle velocities and positions using Eqs. (1) and (2), we need descriptions of $u_{i}^{0}(\boldsymbol{x}), \epsilon(\boldsymbol{x})$, and $a_{i}^{\prime}\left(\boldsymbol{v}^{\prime}, \boldsymbol{x}\right)$. Descriptions of the mean Eulerian velocity in wall-induced turbulence have been obtained theoretically and experimentally [2]. For the mean energy dissipation rate, we can use the asymptotic equality of energy dissipation and energy production [12]. What is unknown in the Langevin equation is the damping function $a_{i}^{\prime}\left(\boldsymbol{v}^{\prime}, \boldsymbol{x}\right)$. The form of the damping function has been established only for homogeneous isotropic turbulence [7], [13], [14]. A well-founded unique formulation of $a_{i}^{\prime}\left(\boldsymbol{v}^{\prime}, \boldsymbol{x}\right)$ in the practically relevant case of inhomogeneous wall-induced turbulence does not exist. We derive such a formula in the next section.

\section{Spatial distribution of the damping function}

The description of the statistics of particle velocities and positions using Eqs. (1) and (2) is Lagrangian. Considerable progress has recently been made in the experimental and numerical determination of the Lagrangian properties of turbulent flow [15]. The results tend to confirm the Lagrangian version of Kolmogorov's universal theory of small scales (see, e.g., [6]). As such, they support the correctness of using the forcing term in Langevin equation (1), which summarizes the effect of the small "viscous" scales on velocity statistics for Re $\gg 1$. But the reported Lagrangian-based results are less useful for specifying the damping term, which significantly determines the statistical behavior of particle velocities and displacements. This behavior is governed by the large "energetic" scales of turbulence, whose structure is inhomogeneous and depends on the configuration in which the turbulent flow occurs. Lagrangian-based statistics can be used to determine the damping function if they encompass a region that is large enough to capture the spatial dependence of the statistical parameters. Furthermore, in the present case, they should apply to configurations relevant to turbulence generated by mean flows along walls. In Sec. 8, we use the few available results satisfying these criteria.

A more rewarding approach for obtaining information leading to a specification of the damping function is to establish a connection with the more explored field of Eulerian (i.e., fixed-point) velocity statistics. This connection is obtained from the Eulerian interpretation of the Fokker-Planck equation associated with Eqs. (1) and (2), also known as the well-mixed condition [16]. It yields a prescription for the probability density function of the fixed-point Eulerian velocity. The Eulerian version of the Fokker-Planck equation associated with Eqs. (1) and (2) and appropriate for wall-induced turbulence is

$$
-\frac{\partial}{\partial u_{i}^{\prime}} a_{i}^{\prime}\left(\boldsymbol{u}^{\prime}, x_{2}\right) p+\frac{1}{2} C_{0} \epsilon \frac{\partial^{2} p}{\partial u_{i}^{\prime} \partial u_{i}^{\prime}}=u_{2}^{\prime} \frac{\partial p}{\partial x_{2}}
$$

where $p=p\left(\boldsymbol{u}^{\prime}\right)$ is the probability density of the fixed-point fluctuating (Eulerian) fluid velocity $\boldsymbol{u}$. Here and hereafter, repeated indices imply summation. In formulating Eq. (3), we use the properties of wall-induced turbulence that the Eulerian-based statistics are stationary $(\partial p / \partial t=0)$ and only vary with the distance $x_{2}$ perpendicular to the wall. This is exactly true for developed turbulent flow in pipes and (almost) twodimensional channels and is a good approximation for turbulent boundary layers along flat plates including neutrally stratified atmospheric surface layers (see, e.g., [2]). While $x_{2}$ is the coordinate perpendicular to both the wall and the mean flow, the coordinates $x_{1}$ and $x_{3}$ are respectively taken parallel to both the mean flow and the wall and perpendicular to the mean flow and parallel to the wall. The mean flow in Eq. (2) is described by

$$
u_{i}^{0}(\boldsymbol{x})=u_{1}^{0}\left(x_{2}\right) \delta_{1 i}
$$

where $\delta_{1 i}$ is the Kronecker delta. 
To specify the damping function $a_{i}^{\prime}\left(\boldsymbol{v}^{\prime}, \boldsymbol{x}\right)$ in Langevin equation (1), we first focus our attention on the inertial sublayer, the region outside the viscous layer and where the mean flow $u_{1}^{0}\left(x_{2}\right)$ can be described by the logarithm law [2]. The inertial sublayer is the domain $\mathrm{Re}^{-1} \ll x_{2} / L \ll 1$, in practice, the domain $30 \mathrm{Re}^{-1} \lesssim$ $x_{2} / L \lesssim 0.1$. Below (see Sec. 7 ), we extend our results to the entire main section $30 \operatorname{Re}^{-1} \lesssim x_{2} / L \leq 1$. In the inertial sublayer, the statistical values of fluctuating velocities can be considered independent of $x_{2}$. Furthermore, an asymptotic analysis of the energy balance equations indicates that dissipation equals production in the inertial sublayer [12]. This implies that in the inertial sublayer, $\epsilon$ behaves according to

$$
\epsilon=\kappa^{-1} \frac{u_{*}^{3}}{x_{2}}, \quad \operatorname{Re}^{-1} \ll \frac{x_{2}}{L} \ll 1,
$$

where $\kappa$ is Von Kármán's constant, $\kappa \approx 0.4$. Meaningful expressions for $p$ are obtained from Eq. (3) only if both the damping term and the diffusion term are retained under the limit process $x_{2} \rightarrow 0$ appropriate for the inertial sublayer. At the same time, $p$ should become independent of $x_{2}$ because the statistical values of fluctuating velocities are constant in the inertial sublayer. This requires that

$$
a_{i}^{\prime}\left(\boldsymbol{u}^{\prime}, x_{2}\right)=\frac{\widetilde{a_{i}^{\prime}}\left(\boldsymbol{u}^{\prime}\right)}{x_{2}}
$$

and Eq. (3) then becomes

$$
-\frac{\partial}{\partial u_{i}^{\prime}} \widetilde{a_{i}^{\prime}}\left(\boldsymbol{u}^{\prime}\right) p+\frac{1}{2} C_{0} \kappa^{-1} u_{*}^{3} \frac{\partial^{2} p}{\partial u_{i}^{\prime} \partial u_{i}^{\prime}}=0 .
$$

Equation (6) specifies the spatial distribution of the damping function. It remains to specify its velocity dependence.

\section{Gaussian velocities and linear damping}

The next step is to assume that the single-point Eulerian velocities have a Gaussian distribution. Measurements indicate typical absolute values of skewness and kurtosis $\lesssim 0.3$ [17]-[19]. In these circumstances, a realistic approach is to use a Langevin model that leads to Gaussian Eulerian velocity statistics. This requires that the function $\widetilde{a_{i}^{\prime}}\left(\boldsymbol{u}^{\prime}\right)$ in $(7)$ be linear in $\boldsymbol{u}^{\prime}$ :

$$
\widetilde{a_{i}^{\prime}}\left(\boldsymbol{u}^{\prime}\right)=-\alpha_{i j} u_{j}^{\prime}
$$

Experimental data indicate that Gaussianity is a reasonable assumption. Linear damping and Gaussianity of the Eulerian velocities can also be inferred from the correspondence between the considered stochastic processes describing velocity fluctuations and the stochastic processes describing closed nondissipative Hamiltonian systems known in theoretical physics. Below (see Sec. 6), we show that such a correspondence exists if the inverse of the Kolmogorov constant is small.

\section{Matching covariances}

Well-mixing is accomplished by requiring that the solutions of Eqs. (7) and (8) comply with the value of the covariance tensor $\sigma_{i j}=\left\langle u_{i}^{\prime} u_{j}^{\prime}\right\rangle$ of the Eulerian velocity field, which we assume to be known (angle brackets denote ensemble means at fixed positions; because we treat stationary turbulence and implement ergodicity, these means can be determined from time averaging at fixed positions). This requirement leads to relations between $\alpha_{i j}$ and $\sigma_{i j}$. These can be obtained by substituting a general three-dimensional Gaussian distribution function in Eq. (7) and equating like terms. It is simpler and more direct to multiply Eq. (7) by $u_{m}^{\prime} u_{n}^{\prime}$, integrate over all values of $\boldsymbol{u}^{\prime}$, and integrate by parts, which yields

$$
\alpha_{n j} \sigma_{j m}+\alpha_{m j} \sigma_{j n}=\delta_{m n} C_{0} \kappa^{-1} u_{*}^{3},
$$


where, we recall, repeated indices imply summation. For the type of turbulence under consideration, we can assume reflectional symmetry with respect to the $x_{1} x_{2}$ plane, i.e., particle velocities in the $x_{3}$ direction are not correlated with those in the streamwise $x_{1}$ and wall-normal $x_{2}$ directions. This implies $\alpha_{13}=\alpha_{31}=\alpha_{23}=\alpha_{32}=0$. The solution of Eq. (9) then becomes

$$
\alpha_{i j}=\frac{1}{2} \kappa^{-1} C_{0} u_{*}^{3}\left(\lambda_{i j}+b_{0} \gamma_{i j}\right)
$$

where $\lambda_{i j}=\sigma_{i j}^{-1}$ is the inverse of the covariance tensor,

$$
\lambda_{i j}=\left(\begin{array}{ccc}
d^{-1} \sigma_{22} & -d^{-1} \sigma_{12} & 0 \\
-d^{-1} \sigma_{12} & d^{-1} \sigma_{11} & 0 \\
0 & 0 & \sigma_{33}^{-1}
\end{array}\right), \quad d=\sigma_{11} \sigma_{22}-\sigma_{12}^{2}>0
$$

We note that $u_{3}^{\prime}$ is not correlated with $u_{1}^{\prime}$ and $u_{2}^{\prime}$. The tensor $\gamma_{i j}$ is defined as

$$
\gamma_{i j}=\left(\begin{array}{ccc}
-d^{-1} \sigma_{12} & d^{-1} \sigma_{11} & 0 \\
-d^{-1} \sigma_{22} & d^{-1} \sigma_{12} & 0 \\
0 & 0 & 0
\end{array}\right) .
$$

In Eq. (10), $b_{0}$ is a constant whose value is unknown; it appears because we have five unknown values of $\alpha_{i j}$ and effectively four equations (see Eqs. (9)) as a result of applying the well-mixed condition. This reflects what is called the nonuniqueness problem: a full statistical specification of fixed-point Eulerian velocities is insufficient for completely specifying the Langevin equation using the well-mixed condition [7]. Equally, because of the phenomenon of convection of small scales by large scales, no relevant information can be obtained from time correlations of Eulerian-based velocities [20]. We now solve the nonuniqueness problem by introducing a reciprocity requirement analogous to the Onsager reciprocity, simultaneously using measurement results and direct numerical simulations (DNSs).

\section{Reciprocity}

We focus our attention on the random process describing fluctuations of fluid particles located at the distance $L_{0}$ from the wall at $t=0$. We introduce dimensionless variables, marked with asterisks, as

$$
x_{2}=L_{0} x_{2}^{*}, \quad t=L_{0} u_{*}^{-1} t^{*}, \quad v_{i}^{\prime}=u_{*} v_{i}^{*}, \quad \alpha_{i j}^{*}=\lambda_{i j}^{*}+b_{0} \gamma_{i j}^{*},
$$

where $\lambda_{i j}^{*}$ and $\gamma_{i j}^{*}$ are defined according to (11) and (12) with $\sigma_{i j}$ replaced with $\sigma_{i j}^{*}=\sigma_{i j} / u_{*}^{2}$. Applying Eq. (13) and dropping the asterisks, from Eqs. (1), (5), (6), (8), and (10), we obtain the dimensionless Langevin equation

$$
\frac{d v_{i}^{\prime}}{d t}=-\frac{1}{2} C_{0} \kappa^{-1}\left(\lambda_{i j}+b_{0} \gamma_{i j}\right) \frac{v_{j}^{\prime}}{x_{2}}+\left(\frac{C_{0} \kappa^{-1}}{x_{2}}\right)^{1 / 2} w_{i}(t),
$$

where

$$
\frac{d x_{2}}{d t}=v_{2}^{\prime}
$$

The damping tensor consists of a symmetric part $\lambda_{i j}$ and an asymmetric part $\gamma_{i j}$. The asymmetric part causes the cross-correlation functions $\overline{v_{1}^{\prime}(0) v_{2}^{\prime}(t)}$ and $\overline{v_{2}^{\prime}(0) v_{1}^{\prime}(t)}$ to differ from each other; the overbar denotes averaging at fixed times over many realizations of the Lagrangian variable, where each realization begins at $t=0$ with a random velocity value chosen in accordance with the Gaussian distribution of the Eulerian 
velocity at the marked point $x_{2}=1$. The difference between the two cross correlations depends on the magnitude of $b_{0}$ : the larger $b_{0}$ is, the larger the difference. The Onsager reciprocity relation [21], [22] implies that the two correlations must be the same: $b_{0}=0$. But the prerequisite for the reciprocity is Hamiltonian dynamics of the particles. Fluid particle dynamics are non-Hamiltonian because they undergo dissipation by viscosity. The viscosity effect appears at the small "viscous" scales of turbulence. The contribution of these scales to the velocity correlation functions vanishes as the value of the Reynolds number increases. More precisely, its effect shrinks to a discontinuity in the slope of the autocorrelation functions as $t \rightarrow 0$ (also see Fig. 1). Nevertheless, the dissipation effect in the energy equation for the turbulent flow does not vanish as $\operatorname{Re} \rightarrow \infty$ [11]. There is a continuous flow of energy from production at large scales to dissipation at small scales. This can be seen in the mean energy dissipation rate, whose magnitude equals $\kappa^{-1} u_{*}^{3} L_{0}^{-1} x_{2}^{-1}$ (see Eq. (5)). The total energy change as a function of the previously defined dimensionless time (see Eq. (13)) amounts to $u_{*}^{2} \kappa^{-1} t x_{2}^{-1}$. Hence, a relative energy change $\Delta H / u_{*}^{2}$ of the order $O(1)$ occurs over a time period $t$ of the order $O(1)$.

There is still a possibility to apply reciprocity to the turbulence problem to a certain degree because the Kolmogorov constant in the Langevin equation has a relatively large value. For flow with a large Reynolds number, the quantity $C_{0}$ can be as large as 6 [14]. It can be seen from Eq. (14) that the time of random velocity fluctuations scales as $C_{0}^{-1}$ and velocities decorrelate over a time $C_{0}^{-1}$. If we describe the solution of the Langevin equation as an expansion in powers of $C_{0}^{-1}$, then the dissipation effect vanishes in the first term. To consider this in more detail, we introduce the expansion parameter

$$
\varepsilon_{1}=C_{0}^{-1}
$$

With time and position scaled as

$$
t=\varepsilon_{1} t^{\prime}, \quad x_{2}=1+\varepsilon_{1} x_{2}^{\prime},
$$

Langevin equation (14) becomes

$$
\frac{d v_{i}^{\prime}}{d t^{\prime}}=-\frac{1}{2} \kappa^{-1}\left(\lambda_{i j}+b_{0} \gamma_{i j}\right) \frac{v_{j}^{\prime}}{1+\varepsilon_{1} x_{2}^{\prime}}+\left(\frac{\kappa^{-1}}{1+\varepsilon_{1} x_{2}^{\prime}}\right)^{1 / 2} w_{i}\left(t^{\prime}\right)
$$

while (cf. Eq. (15))

$$
x_{2}^{\prime}=\int_{0}^{t^{\prime}} v_{2}^{\prime} d t
$$

The total energy change with an error of the order $O\left(\varepsilon_{1}^{2}\right)$ becomes

$$
\frac{\Delta H}{u_{*}^{2}} \sim \varepsilon_{1} t^{\prime}
$$

where $\sim$ means asymptotically equal.

We now consider the probability density and the correlation function of the particle velocity described by Langevin equation (18). Representing the solution as an expansion in powers of $\varepsilon_{1}$, we obtain the first term of such an expansion from Eq. (18) with $\varepsilon_{1}=0$. As a solution, the Langevin equation thus obtained has an exponentially correlated Gaussian process [4], which, as is known, is used to describe closed nondissipative systems [22]. Furthermore, the underlying fluid particle dynamics in the case $\varepsilon_{1}=0$ with $t^{\prime}=O(1)$ are time-invariant Hamiltonian dynamics, which follows from Eq. (20) with $\varepsilon_{1}=0$. This also complies with the dynamics in the intermediate range of times, large compared with the Kolmogorov 

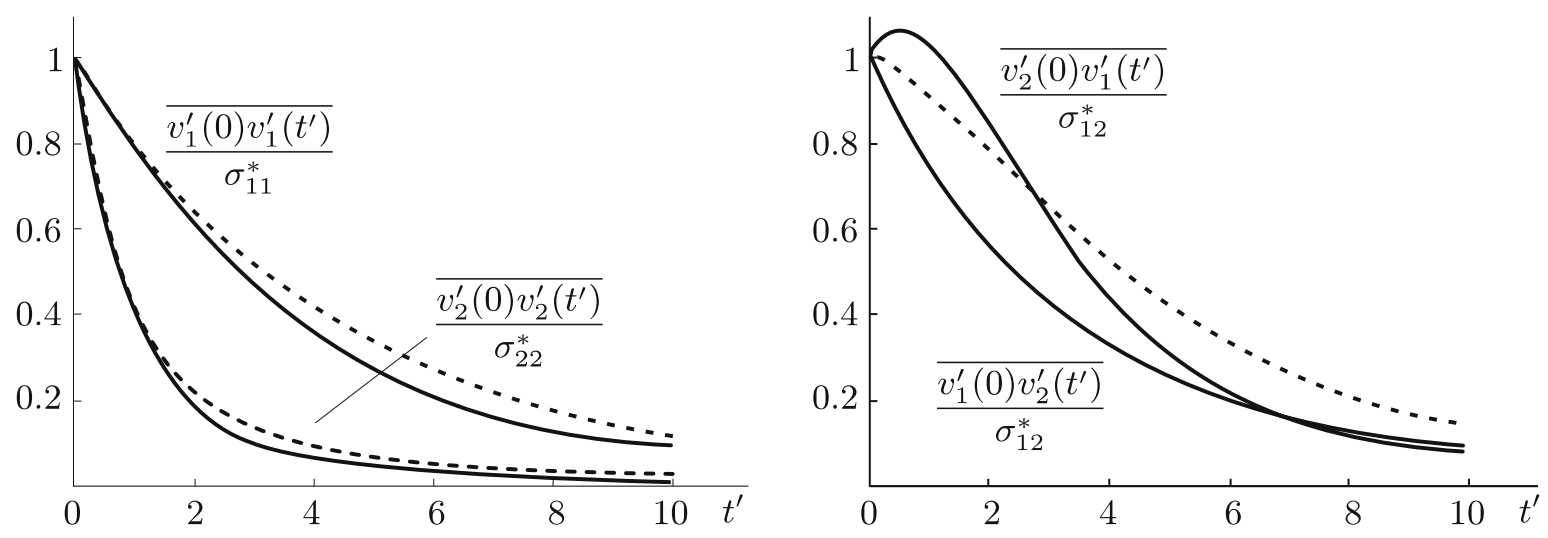

Fig. 1. Lagrangian velocity correlation functions versus the scaled time $t^{\prime}$ for wall-induced turbulence with $b_{1}=1$ and $C_{0}=5$ (solid lines) and nondissipative classical systems (dotted lines); in both cases, $\kappa=0.4, \sigma_{11}=5.67 u_{*}^{2}, \sigma_{22}=1.32 u_{*}^{2}$, and $\sigma_{12}=-u_{*}^{2}$.

"viscous" time and small compared with the energy production time scale, where parcel dynamics based on Hamiltonian descriptions of fluid flow apply (see, e.g., [23]). In the leading order in $\varepsilon_{1}$, the random process describing turbulence is identical to the process describing classical systems. The Onsager reciprocity must hold, and the damping function must be symmetric, $b_{0}=0$ up to $O\left(\varepsilon_{1}^{0}\right)$. Clearly, this is not necessarily the case in an arbitrary order $O\left(\varepsilon_{1}\right)$ : the energy change over the relevant time scale can then no longer be disregarded. We therefore conclude that

$$
b_{0}=b_{1} \varepsilon_{1}
$$

where $b_{1}$ is of the unit order of magnitude. In Sec. 8, we estimate its value from DNS and measurement results.

In Fig. 1, we present the correlation functions of particle velocity versus the dimensionless time $t^{\prime}$ in both the case of a classical system $\left(\varepsilon_{1}=0\right.$ in Eqs. (18)-(21)) and a typical case of wall-induced turbulence $\left(\varepsilon_{1}=0.20, b_{1}=1\right)$. The values for $\kappa$ and $\sigma_{i j}^{*}$ in both cases are $\kappa=0.4, \sigma_{11}^{*}=5.67, \sigma_{22}^{*}=1.32$, and $\sigma_{12}^{*}=-1$. These values agree with measured values in wall-induced turbulence at a high Reynolds number [24]-[26] and with DNS results [27].

We obtained the presented results by numerical time-domain simulation of the coupled fluctuation equations for $v_{1}^{\prime}(t)$ and $v_{2}^{\prime}(t)$ (see Eq. (18)). Particles start at zero at $t=0$ and have initial velocities randomly chosen according to the Gaussian Eulerian velocity distribution at that point (passive marking of fluid particles). We simulated the velocities of $10^{6}$ particles. In the case of wall-induced turbulence, we assumed that particles are reflected at the wall: $x_{2}^{\prime}=-\varepsilon_{1}^{-1}$. We took the coefficient $\left(1+\varepsilon_{1} x_{2}^{\prime}\right)^{-1}$ to be constant and equal to $\delta^{-1}$ in a small region near the wall $0 \leq 1+\varepsilon_{1} x_{2}^{\prime} \leq \delta, \delta \ll 1$. The results shown in Fig. 1 and elsewhere in this paper correspond to $\delta=10^{-3}$. We found that reducing the value of $\delta$ had a negligible effect on the results if the time step length in the numerical solution routine was shortened accordingly. This illustrates that the presence of a small viscous layer at the wall affects the results weakly and also shows that the presence of terms like $\left(1+\varepsilon_{1} x_{2}^{\prime}\right)^{-1}$ in the fluctuation equations does not lead to singular behavior.

It can be seen that the contribution of terms of the order $O\left(\varepsilon_{1}\right)$ is relatively small. The effect of inhomogeneity due to the term $\left(1+\varepsilon_{1} x_{2}^{\prime}\right)^{-1}$ in the Langevin equation is modest on the small time scale of velocity fluctuations and reduces further as $\varepsilon_{1}$ decreases or $C_{0}$ increases. Particle dispersion is manifested on a much longer time scale, and the inhomogeneity due to the $x_{2}$ dependence in the coefficients of the Langevin equation here has an overwhelming effect on the statistics. Such behavior can be accurately 
assessed by simulating the presented equations including integrating the time records of velocity to calculate displacements. In Sec. 9, we demonstrate similar behavior analytically using closed-form expressions appropriate for the diffusion limit.

\section{Generalization to the main section}

Up to now, our attention was focused on the damping function for the inertial sublayer. Away from the wall and the inertial sublayer, in other words, in the main section $x_{2} / L=O(1)$, the statistical means of fluctuating Eulerian velocities can no longer be assumed constant. A straightforward way to extend the previously obtained expressions for the damping function to the main section is to take the tensors $\lambda_{i j}$ and $\gamma_{i j}$ specified by Eqs. (11) and (12) to depend on $x_{2}, \lambda_{i j}=\lambda_{i j}\left(x_{2}\right)$ and $\gamma_{i j}=\gamma_{i j}\left(x_{2}\right)$, via the dependence of $\sigma_{i j}$ on $x_{2}$. We note that the energy dissipation rate $\epsilon=\epsilon\left(x_{2}\right)$ is no longer approximated according to Eq. (5), and well-mixing is based on Eq. (3) instead of (7). Langevin equation (14) can then be written in the dimensional form

$$
\frac{d v_{i}^{\prime}}{d t}=-\frac{1}{2}\left(C_{0} \lambda_{i j}+b_{1} \gamma_{i j}\right) \epsilon v_{j}^{\prime}+\phi_{i}+\sqrt{C_{0} \epsilon} w_{i}(t),
$$

while particle positions can be described by (cf. Eqs. (2) and (4))

$$
\frac{d x_{i}}{d t}=u_{1}^{0}\left(x_{2}\right) \delta_{i 1}+v_{i}^{\prime}
$$

A term $\phi_{i}=\phi_{i}\left(\boldsymbol{v}, x_{2}\right)$ describing the drift due to spatial changes of covariances is included in Eq. (22). Expressions for $\phi_{i}$ can be derived from the well-mixing condition, which corresponds to Eq. (3). Requiring the well-mixing of first-order moments, i.e., multiplying Eq. (3) by $u_{j}^{\prime}$, integrating over the entire $\boldsymbol{u}^{\prime}$ domain, and integrating by parts, we obtain

$$
\phi_{i}=\frac{d \sigma_{i 2}}{d x_{2}} .
$$

If we require Gaussianity of the fixed-point Eulerian velocities throughout the main section, then we can describe the drift term according to Thomson as (see [16])

$$
\phi_{i}=\frac{d \sigma_{i 2}}{d x_{2}}+\frac{1}{2} \lambda_{j m} \frac{d \sigma_{i j}}{d x_{2}}\left(v_{2}^{\prime} v_{m}^{\prime}-\sigma_{2 m}\right)
$$

But this formulation is not unique; other forms containing a quadratic relation for the velocities are possible, for example, the Borgas model [28]. The coefficients in all these formulations are proportional to the slope of the covariances, and all these formulations satisfy the well-mixing condition. Nevertheless, the nonuniqueness problem for $\phi_{i}$ is of the second order: the ultimate effect of the covariance inhomogeneity is limited because the terms containing $C_{0} \epsilon$ in Eq. (22) are relatively large. For sufficiently large Re, we can identify an inertial sublayer. Here, $\epsilon$ is very large (see Eq. (5)), and the contribution from the covariance inhomogeneity via $\phi_{i}$ is very small. In this region, Eq. (22) is well described by Eq. (14) and becomes identical to (14) if we pass to the limit of the inertial sublayer. In this case, the drift term vanishes. In the main section, $\epsilon$ decreases as the distance from the wall increases (see Eq. (5)), but the covariance inhomogeneity effect remains small because the numerical value of $C_{0}$ is rather large.

In summary, the contribution of the terms describing drift due to the covariance inhomogeneity is small. Compared with the leading terms in the damping function, the magnitude of the covariance inhomogeneity terms scales as $C_{0}^{-1} x_{2} L^{-1}$, where $x_{2}$ is the distance from the wall and $\mathrm{L}$ is the external length, i.e., the pipe radius, channel half-width, or turbulent boundary layer thickness along a wall or along the earth's surface.

While the contribution of the drift term $\phi_{i}$ is generally small, the contribution of the difference between the two expressions for $\phi_{i}$, denoted by $\Delta \phi_{i}$, is even smaller. At the marked point, the particle velocity 
probability distribution equals that of the Eulerian velocity. The mean and standard deviation of $\Delta \phi_{i}$ are then equal to zero. It is only at long times that statistical means of $\Delta \phi_{i}$ become nonzero. In Sec. 9, we present statistical descriptions of $\boldsymbol{v}^{\prime}$ valid in the long-time limit, i.e., the diffusion limit. They reveal differences between the statistical values of the particle velocity and of the Eulerian velocity, which are small. The net effect is that the contribution to the particle dispersion from the terms relating to $\Delta \phi_{i}$ is very small. Numerical simulations reveal differences between the statistical values of particle displacement using Eqs. (24) and (25) that are not larger than 2\%. Dispersion predictions based on either (24) or (25) are equally good.

The linearly varying part of the damping function is the important term in Eq. (22). It consists of a symmetric part and an asymmetric part. The symmetric part constitutes the leading term in the expansion

in $C_{0}^{-1}$. This symmetric part is unique. It is completely specified over the entire main section, i.e., no other forms are possible, if we require the following for the formulation in the leading order in $C_{0}$ : Gaussianity of the fixed-point Eulerian velocities, well-mixing according to Eq. (3), and reciprocity analogous to the Onsager reciprocity. Asymmetry can occur in the damping only in terms of the order $O\left(C_{0}^{-1}\right)$ compared with the leading terms. Below, we establish the value of the parameter $b_{1}=b_{1}\left(x_{2}\right)$ in these terms.

\section{The value of the asymmetry parameter $b_{1}$}

We focus our attention on the (Lagrangian) correlation between the velocity of a passively marked fluid particle and its velocity at $t=0$ at the position $x_{2}=x_{20}$. Multiplying Eq. (22) by $v_{k}^{\prime}(0)$, averaging, using the Markov property, expanding for small times $t>0$, applying Gaussianity in the case of Eq. (25), and using Eqs. (11) and (12), we obtain

$$
\frac{d\left(\overline{v_{k}^{\prime}(0) v_{i}^{\prime}(t)}\right)}{d t}=-\frac{1}{2} C_{0} \epsilon \delta_{i k}-\frac{1}{2} b_{1} \epsilon\left(\delta_{i 1} \delta_{k 2}-\delta_{i 2} \delta_{k 1}\right)
$$

up to $O(t)$. From this expression, we obtain the relation for the cross-correlation functions up to $O(t)$,

$$
\frac{d\left(\overline{v_{1}^{\prime}(0) v_{2}^{\prime}(t)}\right)}{d t}=-\frac{d\left(\overline{v_{2}^{\prime}(0) v_{1}^{\prime}(t)}\right)}{d t}=\frac{1}{2} b_{1} \epsilon
$$

It can be seen that the value of $b_{1}$ can be obtained directly from this relation. Estimates of the cross correlations in developed turbulent flow in a pipe of radius $R_{0}$ at various radial positions and for $\operatorname{Re}=$ $u_{*} R_{0} / \nu=320$ were recently obtained using three-dimensional particle tracking velocimetry [29]. We show results from [29] in Fig. 2 and present values for $b_{1}$ deduced from these results as a function of the distance from the wall in Fig. 3.

We estimated the slopes of cross correlations from the experimental data and obtained values for $\epsilon$ by DNS of the same configuration. We also used the experimental results to estimate the value of the Kolmogorov constant from the Lagrangian velocity structure function [29]. We thus determined the Kolmogorov constants appropriate for three directions and the difference between these values indicates the magnitude of the anisotropy. The degree of anisotropy determined by the differences between the Kolmogorov constants in the streamwise and wall-normal directions $C_{1}$ and $C_{2}$ as a function of the wall distance is also shown in Fig. 3. It can be seen that the isotropy is maximum in the interior of the pipe. Here, the Reynolds number corresponding to the distance $u_{*} x_{2} / \nu$ from the wall is largest, and a state of isotropy of the small scales in accordance with Kolmogorov theory becomes visible. From the results at a smaller Reynolds number $\left(u_{*} R_{0} / \nu=180\right)$, we learned that the isotropy degree increases as Re increases [29] and the values of $b_{1}$ overall decrease by approximately $15 \%$ over the entire radius. We can conclude that 


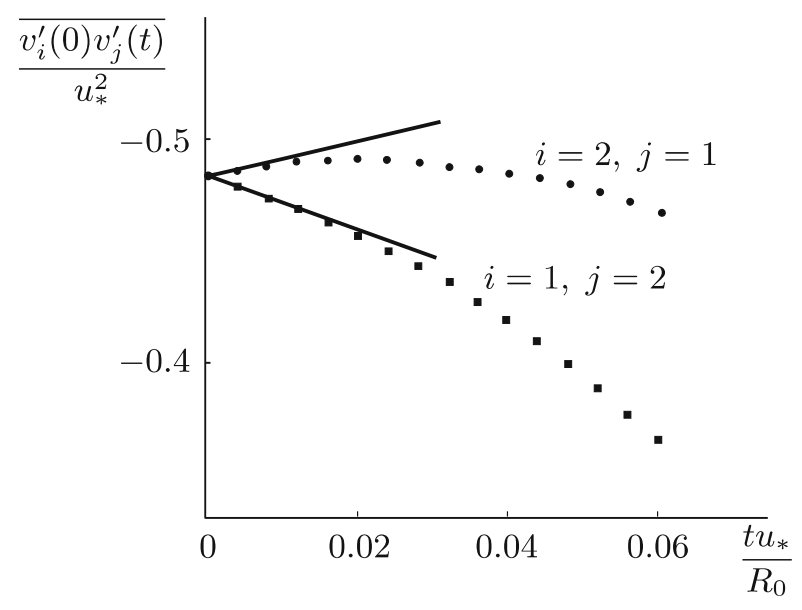

Fig. 2. Cross correlations versus time (dots) and their slopes (straight lines) measured by threedimensional particle tracking in developed turbulent flow in a pipe of radius $R_{0}$ for $x_{20}=0.5 R_{0}$ and $\operatorname{Re}=u_{*} R_{0} / \nu=320[29]$.

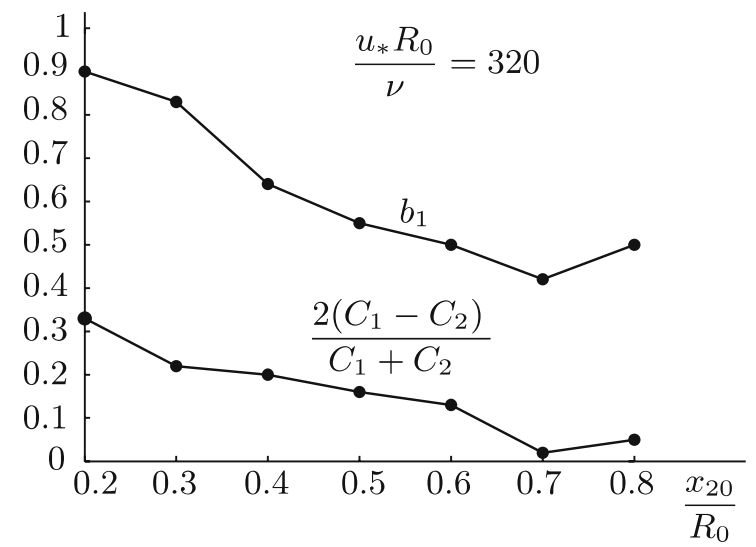

Fig. 3. The asymmetry coefficient $b_{1}$ and anisotropy degree $2\left(C_{1}-C_{2}\right) /\left(C_{1}+C_{2}\right)$ inferred from the measurements in [29].

the value of the asymmetry parameter $b_{1}$ is less than unity in wall-induced turbulence at a high Reynolds number.

Interestingly, cross-correlation functions do not seem to be affected by small "viscous" scales as $t \rightarrow 0$, where $\tau_{\eta}=\sqrt{\nu / \epsilon}$ is the Kolmogorov "viscous" time. While slopes of autocorrelations tend to zero as $t \rightarrow 0$, such behavior is not apparent in the cross correlations. This can be ascribed to the isotropy of viscous forces: the cross correlations of viscous forces are zero. Because of this behavior, we can rather precisely determine the values of $b_{1}$ from measured cross-correlations, even at not very high values of the Reynolds number. The experimentally determined slopes of the two relevant cross correlations also consistently showed the mutual antisymmetry predicted by Eq. (27) and also seen in Fig. 2. The cross correlations derived from the DNSs, on the other hand, showed some deviations from the experimentally observed antisymmetric behavior at certain radial positions. This may be due to an apparent anisotropic damping as a result of approximations in the numerical integration schemes. Alternatively, some anisotropic behavior of viscous forces present at a finite Reynolds number may have been filtered out during the measurements. In any case, these differences are too small to affect the conclusions regarding the value of $b_{1}$. 


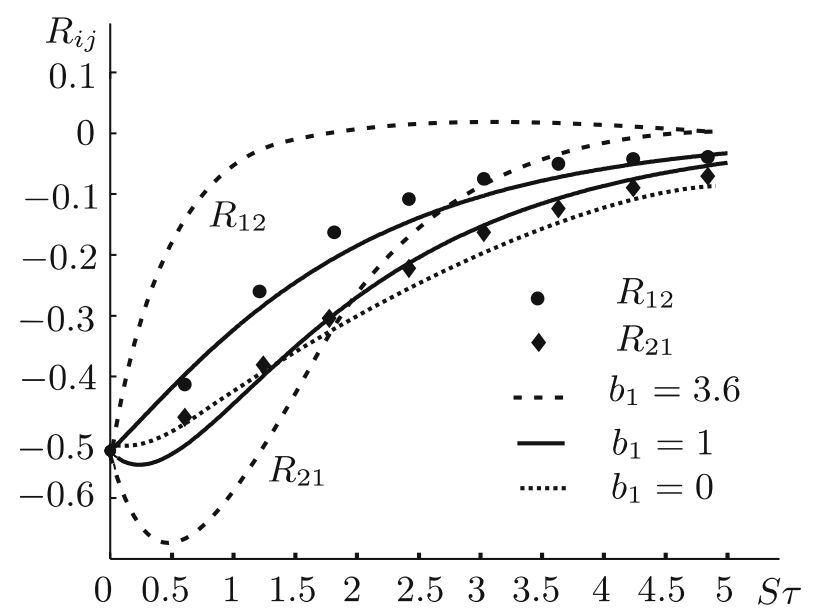

Fig. 4. The cross correlations $R_{i j}$ of the $i$ th and $j$ th velocity components as functions of the dimensionless time lag in [30]: • and $\bullet$ are the DNS results for the Lagrangian statistics, the dashed lines are the results of calculating with the Borgas model $\left(b_{1}=3.6\right)$, the dotted lines are the results of calculating with the Thomson model $\left(b_{1}=0\right)$, and the solid lines are the results of calculating with our model $\left(b_{1}=1\right)$ with the parameter values $\sigma_{11}=0.57, \sigma_{22}=0.31, \sigma_{12}=-0.22, d \sigma_{i j} / d x_{2}=0$, $\epsilon=0.66$, and $C_{0}=4$.

A value for $b_{1}$ can also be deduced from the difference in the cross-correlation functions inferred from DNSs of homogeneous turbulence in uniform shear flow at a Taylor Reynolds number near $150(\operatorname{Re} \approx$ 1500) [30]. Interestingly, the value of $b_{1}$ that best fits the cross correlations is approximately 1 (see Fig. 4). In [30], the results were also compared with stochastic models proposed by Thomson and Borgas. The Thomson model amounts to complete symmetry $\left(b_{1}=0\right)$, while the Borgas model exaggerates asymmetry with a value of $b_{1} \approx 3.6$ (see Fig. 4). The nonstationarity effects apparent in uniform shear flow are minor and can be disregarded on the time scale of velocity correlations.

In general, we can conclude that the asymmetry of the damping term is relatively small. Because reciprocity must hold for terms of the order $O\left(C_{0}^{1}\right)$, asymmetry only appears in terms of the order $O\left(C_{0}^{0}\right)$ (see Eq. (22)). The value of the asymmetry constant $b_{1}$ is less than unity. The asymmetry effect is noticeable in the difference between the cross-correlation functions of particle velocity (see Fig. 1). The statistical values of particle displacement, on the other hand, vary only very slightly with $b_{1}$ for $\left|b_{1}\right| \lesssim 1$. This is illustrated in Fig. 5, where we show the wall-normal distribution of particles in the logarithm layer as a function of horizontal distance considering a point source at $\left(x_{1}, x_{2}\right)=\left(0, L_{0}\right)$. The curves shown correspond to the $x_{2}$ positions with the nonexceedance probabilities $16 \%, 50 \%$, and $84 \%$. We show the results for the cases $b_{1}=0$ and $b_{1}=1$ with $C_{0}=5.5$. The distribution of the mean horizontal (wind) velocity agrees with the logarithm law $u_{1}^{0}\left(x_{2}\right)=\kappa^{-1} \log \left(9 \operatorname{Re}_{0} x_{2} / L_{0}\right)$, where $\operatorname{Re}_{0}=u_{*} L_{0} \nu^{-1}$; we set $\operatorname{Re}_{0}=3 \cdot 10^{4}$ in the simulations. The values of other parameters in the Langevin model correspond to those in Fig. 1. It can be seen that the "plume" of particles evolving from the point source hardly differs for $b_{1}=0$ and $b_{1}=1$. In the next section, we explain and generalize this result based on analytic expressions for long-time displacement statistics. The analytic expressions yield typical changes of at most $3 \%$ in dispersion parameters as $b_{1}$ increases from 0 to 1 . We conclude that the uncertainty in the value of $b_{1}$ and in the specification of $\phi_{i}$ (cf. Eqs. (24) and (25)) hardly affect the dispersion predictions. For Re $\gtrsim 2000$, there is a considerable distinction between the various turbulence scales, and the value of $C_{0}$ reaches its asymptotic limit of approximately 6 . We then expect predictions based on Eqs. (22)-(24) with $b_{1}=0$ to be quite accurate; this category includes many applications. 


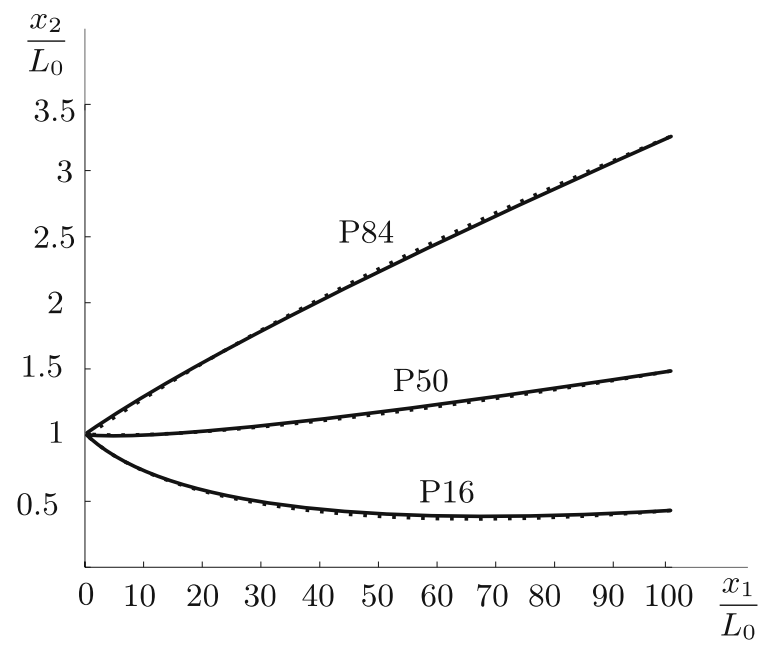

Fig. 5. The wall-normal particle distribution versus horizontal (streamwise) position due to a point source for values of the asymmetry parameter $b_{1}=0$ (solid lines) and $b_{1}=1$ (dotted lines): the $x_{2}$ positions with the nonexceedance probabilities $16 \%$ (P16), 50\% (P50), and 84\% (P84).

\section{The diffusion limit}

Numerical simulation using the Langevin equation yields data for the particle velocity, and integrating yields data for the particle displacement. Dispersion statistics are obtained by averaging these data. A more direct way to obtain information about the statistics of particle displacement is to analyze the behavior of the Langevin equation at long times after particle marking. The long-time behavior is called the diffusion limit, which we consider in what follows.

For convenience in algebraic manipulations, we introduce the particle displacement $\boldsymbol{x}^{\prime}$ relative to displacement by the mean velocity. Instead of Eq. (23), we have

$$
\frac{d x_{i}^{\prime}}{d t}=v_{i}^{\prime}
$$

The Fokker-Planck equation for the time-dependent joint probability density $p=p\left(\boldsymbol{v}^{\prime}, \boldsymbol{x}^{\prime}, t\right)$ of the particle velocity and particle position described by fluctuation equations (22) and (28) can be formulated as [31]

$$
\frac{\partial p}{\partial t}=\frac{1}{2} \epsilon C_{0}\left\{\frac{\partial}{\partial v_{i}^{\prime}}\left(\alpha_{i j} v_{j}^{\prime} p\right)+\frac{\partial^{2} p}{\partial v_{i}^{\prime} \partial v_{i}^{\prime}}\right\}-\frac{\partial}{\partial v_{i}^{\prime}}\left(\phi_{i} p\right)-\frac{\partial}{\partial x_{i}^{\prime}}\left(v_{i}^{\prime} p\right)
$$

where

$$
\alpha_{i j}=\lambda_{i j}+C_{0}^{-1} b_{1} \gamma_{i j} .
$$

To study the long-time behavior of solutions of (29), we must couple time to some small dimensionless number. Ideally, this number tends to zero in some limit process like the inverse of the Reynolds number. But such a parameter is absent from (29). In the general case, there is no limit process by which the diffusion limit of inhomogeneous turbulence becomes exact [32]. All that remains is to develop a diffusion limit based on the smallness of $C_{0}^{-1}$. We hope that the contribution of the truncated higher-order terms is small although the value of $C_{0}^{-1}$ is finite. At the end of this section, we compare the results in the diffusion limit thus obtained with the results of numerical simulations of the Langevin equation. The diffusion limit is found to work reasonably well. 
The adopted expansion procedure is similar to the methods van Kampen used to analyze the Kramers equation in [4]. Time is scaled as $t=C_{0} t^{\prime}$, and Eq. (29) then becomes

$$
\frac{\partial}{\partial v_{i}^{\prime}}\left(\alpha_{i j} v_{j}^{\prime} p\right)+\frac{\partial^{2} p}{\partial v_{i}^{\prime} \partial v_{i}^{\prime}}=2 \epsilon^{-1} C_{0}^{-1}\left\{\frac{\partial}{\partial v_{i}^{\prime}}\left(\phi_{i} p\right)+\frac{\partial}{\partial x_{i}^{\prime}}\left(v_{i}^{\prime} p\right)\right\}+2 \epsilon^{-1} C_{0}^{-2} \frac{\partial p}{\partial t^{\prime}} .
$$

Its solution can be written as an expansion in powers of $C_{0}^{-1}$ :

$$
p=p_{0}+C_{0}^{-1} p_{1}+C_{0}^{-2} p_{2}
$$

Substituting this expression in (31) and equating like powers of $C_{0}$ yields the equations

$$
\begin{aligned}
& \frac{\partial}{\partial v_{i}^{\prime}}\left(\alpha_{i j} v_{j}^{\prime} p_{0}\right)+\frac{\partial^{2} p_{0}}{\partial v_{i}^{\prime} \partial v_{i}^{\prime}}=0, \\
& \frac{\partial}{\partial v_{i}^{\prime}}\left(\alpha_{i j} v_{j}^{\prime} p_{1}\right)+\frac{\partial^{2} p_{1}}{\partial v_{i}^{\prime} \partial v_{i}^{\prime}}=2 \epsilon^{-1}\left\{\frac{\partial}{\partial v_{i}^{\prime}}\left(\phi_{i} p_{0}\right)+\frac{\partial}{\partial x_{i}^{\prime}}\left(v_{i}^{\prime} p_{0}\right)\right\}, \\
& \frac{\partial}{\partial v_{i}^{\prime}}\left(\alpha_{i j} v_{j}^{\prime} p_{2}\right)+\frac{\partial^{2} p_{2}}{\partial v_{i}^{\prime} \partial v_{i}^{\prime}}=2 \epsilon^{-1}\left\{\frac{\partial}{\partial v_{i}^{\prime}}\left(\phi_{i} p_{1}\right)+\frac{\partial}{\partial x_{i}^{\prime}}\left(v_{i}^{\prime} p_{1}\right)\right\}+2 \epsilon^{-1} \frac{\partial p_{0}}{\partial t^{\prime}} .
\end{aligned}
$$

The solution of (32) matching the distribution of the fixed-point Eulerian velocity at the instant of passive marking is

$$
p_{0}(\boldsymbol{x}, \boldsymbol{v}, t)=G_{0}(\boldsymbol{x}, t)(2 \pi)^{3 / 2} \lambda^{1 / 2} e^{-\lambda_{i j} v_{i}^{\prime} v_{j}^{\prime} / 2},
$$

where $\lambda$ is the determinant of $\lambda_{i j}$; we note that $\partial\left(\gamma_{i j} v_{j}^{\prime} p_{0}\right) / \partial v_{i}^{\prime}=0$. The function $G_{0}(\boldsymbol{x}, t)$ describes the distribution of the particle position or, equivalently, the distribution of the concentration of a passively added admixture:

$$
G_{0}(\boldsymbol{x}, t)=\int_{-\infty}^{+\infty} p_{0}(\boldsymbol{x}, \boldsymbol{v}, t) d \boldsymbol{v}
$$

The equation for $G_{0}=G_{0}(\boldsymbol{x}, t)$ can be derived from (33) and (34) as follows. A realistic solution for $p_{2}$ is obtained from (34) if the integral of the right-hand side over the entire $\boldsymbol{v}$ domain is zero. Noting that $\phi_{i} p_{1} \rightarrow 0$ as $|\boldsymbol{v}| \rightarrow 0$, we have

$$
-\frac{\partial}{\partial x_{i}} \int_{-\infty}^{+\infty} v_{i}^{\prime} p_{1} d \boldsymbol{v}=\frac{\partial G_{0}}{\partial t}
$$

The integral can be evaluated by multiplying (33) by $v_{k}^{\prime}$ and integrating by parts,

$$
-\alpha_{k j} \int_{-\infty}^{+\infty} v_{j}^{\prime} p_{1} d \boldsymbol{v}=2 \epsilon^{-1}\left\{-\int_{-\infty}^{+\infty} \phi_{k} p_{0} d \boldsymbol{v}+\frac{\partial}{\partial x_{i}^{\prime}} \int_{-\infty}^{+\infty} v_{i}^{\prime} v_{k}^{\prime} p_{0} d \boldsymbol{v}\right\} .
$$

Using Eq. (35) and multiplying by $\alpha_{i k}^{-1}$, we obtain

$$
\int_{-\infty}^{+\infty} v_{i}^{\prime} p_{1} d \boldsymbol{v}=2 \alpha_{i k}^{-1} \epsilon^{-1}\left\{G_{0} \frac{d \sigma_{k 2}}{d x_{2}}-\frac{\partial}{\partial x_{j}}\left(\sigma_{j k} G_{0}\right)\right\}=-2 \alpha_{i k}^{-1} \sigma_{j k} \epsilon^{-1} \frac{\partial G_{0}}{\partial x_{j}^{\prime}} .
$$

It was noted that

$$
\int_{-\infty}^{+\infty} \phi_{i} p_{0} d \boldsymbol{v}=G_{0} \frac{d \sigma_{2 i}}{d x_{2}}
$$

for both descriptions of $\phi_{i}$, Eqs. (24) and (25), because the probability distribution of the particle velocity according to (35) equals that of the Eulerian fixed-point velocity, which results in the mean value of the 
difference between the two expressions vanishing. We can therefore conclude that in the leading order of the diffusion limit, the dispersion results are unaffected by the nonuniqueness of $\phi_{i}$ ! A difference between the mean values of the two expressions for $\phi_{i}$ occurs only in higher orders. Considering higher-order solutions for $p_{1}$ and $p_{2}$, i.e., solutions of (33) and (34) not presented here, we found that the difference between the two mean values of $\phi_{i}$ scales as $C_{0}^{-2}$. Its ultimate contribution to the dispersion is very small and leads to relative deviations in statistical values of the dispersion of a few percent or less for values of $C_{0} \geq 5$.

Substituting (39) in (37) yields the diffusion equation. Converting from the relative position $\boldsymbol{x}^{\prime}$ to $\boldsymbol{x}$ and from the scaled time $t^{\prime}$ to $t$, we obtain

$$
\frac{\partial G_{0}}{\partial t}+u_{1}^{0} \frac{\partial G_{0}}{\partial x_{1}}=\frac{\partial}{\partial x_{i}}\left(D_{i j} \frac{\partial G_{0}}{\partial x_{j}}\right)
$$

where the diffusion coefficient $D_{i j}=D_{i j}\left(x_{2}\right)$ is defined as

$$
D_{i j}=\frac{2 \alpha_{i k}^{-1} \sigma_{j k}}{C_{0} \epsilon}
$$

The applicability of this result is not limited to the symmetric form of the linear damping function in the Langevin equation. The result also applies to asymmetric damping, whose effect appears via the tensor $\alpha_{i j}$ given by (30). We can write

$$
\alpha_{i k}^{-1} \sigma_{j k}=\left(\begin{array}{ccc}
v_{1}^{-1}\left(\sigma_{12}^{2}+\sigma_{11}^{2}\right) & v_{1}^{-1}\left\{\sigma_{12}\left(\sigma_{11}+\sigma_{22}\right)-\frac{b_{1} d}{C_{0}}\right\} & 0 \\
v_{1}^{-1}\left\{\sigma_{12}\left(\sigma_{11}+\sigma_{22}\right)+\frac{b_{1} d}{C_{0}}\right\} & v_{1}^{-1}\left(\sigma_{12}^{2}+\sigma_{22}^{2}\right) & 0 \\
0 & 0 & \sigma_{33}^{2}
\end{array}\right)
$$

where $v_{1}=1+b_{1}^{2} / C_{0}^{2}$ and $d=\sigma_{11} \sigma_{22}-\sigma_{12}^{2}$. Substituting (42) in diffusion equation (40) and rearranging terms gives

$$
\frac{\partial G_{0}}{\partial t}+\left\{u_{1}^{0}-\frac{2}{C_{0}^{2}} \frac{\partial}{\partial x_{2}}\left(v_{1}^{-1} b_{1} d \epsilon^{-1}\right)\right\} \frac{\partial G_{0}}{\partial x_{1}}=\frac{\partial}{\partial x_{i}}\left(D_{i j}^{s} \frac{\partial G_{0}}{\partial x_{j}}\right)
$$

where $D_{i j}^{\mathrm{s}}$ is the symmetric diffusion tensor

$$
D_{i j}^{\mathrm{s}}=\frac{2}{C_{0} \epsilon}\left(\begin{array}{ccc}
v_{1}^{-1}\left(\sigma_{12}^{2}+\sigma_{11}^{2}\right) & v_{1}^{-1} \sigma_{12}\left(\sigma_{11}+\sigma_{22}\right) & 0 \\
v_{1}^{-1} \sigma_{12}\left(\sigma_{11}+\sigma_{22}\right) & v_{1}^{-1}\left(\sigma_{12}^{2}+\sigma_{22}^{2}\right) & 0 \\
0 & 0 & \sigma_{33}^{2}
\end{array}\right)
$$

The asymmetry effect now appears in a drift term in the mean flow direction and in a correction in the diffusivity tensor. The drift term is negligible: drift competes with convection by the mean flow, which is very large in wall-induced turbulence at a large Reynolds number. But the correction in the diffusivity tensor is also very small because it involves the factor $b_{1}^{2} / C_{0}^{2}$. For $b_{1}=1$ and $C_{0}=5.5$, this factor amounts to 0.03 . Deviations in the dispersion statistics due to possible variations of $b_{1}$ between 0 and 1 thus amount to only $3 \%$ ! This shows that the uncertainty due to a possible asymmetry in the damping function is a negligible factor in dispersion. At $b_{1}=0$, the description of the diffusion tensor reduces to

$$
D_{i j}=\frac{2 \sigma_{i k} \sigma_{j k}}{C_{0} \epsilon} .
$$

Substituting this result in (40), we obtain a diffusion equation in which all uncertainty related to nonuniqueness has vanished. Because of the Onsager symmetry, which holds in the leading order in $C_{0}$, the nonuniqueness uncertainty appears only in terms of the relative magnitude $O\left(C_{0}^{-2}\right)$. The contribution of these terms can be disregarded when allowing for errors in the dispersion parameters that are at most a few percent. 
The remaining unanswered question is what the error is in the diffusion approximation because of truncating the expansion in powers of $C_{0}^{-1}$ leading to the diffusion approximation irrespective of the terms related to nonuniqueness. This question can be answered by deriving expressions for the higher-order terms in the expansion. But the expressions seem long and complicated. A more pragmatic approach is to compare the results of the diffusion equation with the results of numerically simulating the Langevin equation. In doing so, we focus attention on the inertial sublayer, where the Fokker-Planck equation reduces to Eq. (14). We must compare the statistics of the particle displacement in the wall-normal direction. These have a primary importance because they also substantially determine the dispersion in the mean flow direction via the $x_{2}$ dependence of the mean flow $u_{1}^{0}\left(x_{2}\right)$. The probability density of particle displacement in the $x_{2}$ direction, denoted by $G_{20}=G_{20}\left(x_{2}\right)$, is defined as

$$
G_{20}\left(x_{2}\right)=\int_{-\infty}^{+\infty} \int_{-\infty}^{+\infty} G_{0}(\boldsymbol{x}) d x_{1} d x_{3}
$$

Integrating (43) over the entire $x_{1}$ and $x_{3}$ domain, taking the inertial sublayer limit, and setting $b_{1}=0$, we obtain

$$
\frac{\partial G_{20}}{\partial t}=\kappa_{1} u_{*} \frac{\partial}{\partial x_{2}}\left(x_{2} \frac{\partial G_{20}}{\partial x_{2}}\right)
$$

where

$$
\kappa_{1}=\frac{2 \kappa}{C_{0}}\left(1+\frac{\sigma_{22}^{2}}{u_{*}^{4}}\right)
$$

and $u_{*}$ is the shear velocity.

Expressions for the statistical moments $\overline{x_{2}^{n}}=\int_{-\infty}^{+\infty} x_{2}^{n} G_{20} d x_{2}$ are obtained by multiplying (45) by $x_{2}^{n}$, integrating over the entire $x_{2}$ domain and integrating the term in the right-hand side by parts. We thus obtain the first cumulant (cumulants are marked by a double overbar).

$$
\overline{\overline{x_{2}^{*}}}=\overline{x_{2}^{*}}=1+\kappa_{1} t^{*}
$$

where $x_{2}^{*}=x_{2} / L_{0}, L_{0}$ is the particle position at $t=0$, and $t^{*}=u_{*} t / L_{0}$. Expressions for higher-order moments can be derived similarly. Using the relations between moments and cumulants [4], we obtain

$$
\overline{\overline{x_{2}^{* 2}}}=\kappa_{1}^{2} t^{* 2}+2 \kappa_{1} t^{*}, \quad \overline{\overline{x_{2}^{* 3}}}=2 \kappa_{1}^{3} t^{* 3}+6 \kappa_{1}^{2} t^{* 2}, \quad \overline{\overline{x_{2}^{* 4}}}=6 \kappa_{1}^{4} t^{* 4}+24 \kappa_{1}^{3} t^{* 3} .
$$

These results exhibit significant values for higher-order cumulants. The long-time limits of skewness $\overline{\overline{x_{2}^{* 3}}}\left(\overline{\overline{x_{2}^{* 2}}}\right)^{-3 / 2}$ and kurtosis $\overline{\overline{x_{2}^{* 4}}}\left(\overline{\overline{x_{2}^{* 2}}}\right)^{-2}$ derived from these expressions are respectively 2 and 6 , which indicates a strong non-Gaussian behavior. This is a consequence of the inhomogeneity of wall-induced turbulence reflected in the change of energy dissipation and diffusivity in the wall-normal direction.

In Fig. 6a, we show $\left(\overline{\overline{x_{2}^{n}}}\right)^{1 / n}$ for $n=1,2,3,4$ obtained by numerically simulating the Langevin equation appropriate for the logarithm layer divided by their respective values according to the diffusion limit (cf. Eqs. (47) and (48)) as a function of the dimensionless time $u_{*} t / L_{0}$. The parameter values used in the comparison are listed in the figure caption. For times shortly after marking, the solutions of the diffusion equation differ considerably. The differences are inherent to the diffusion approximation, which is known to exhibit serious errors in the short-time limit. At long times, the ratios of the cumulants approach constant values, but they are less than unity by an amount that increases as $n$ increases. This illustrates that the diffusion limit never becomes exact in inhomogeneous turbulent flow [32]. Nevertheless, the deviations are not dramatic if we accept some inaccuracy in the tails of the probability distributions. The deviations can be reduced by correcting the value of the diffusivity $\kappa_{1}$ by multiplying it by 0.85 (see Fig. $6 \mathrm{~b}$ ). 


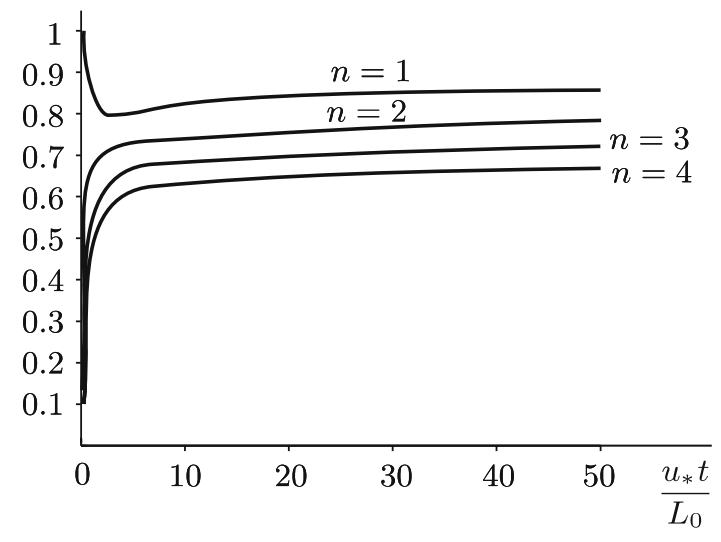

a

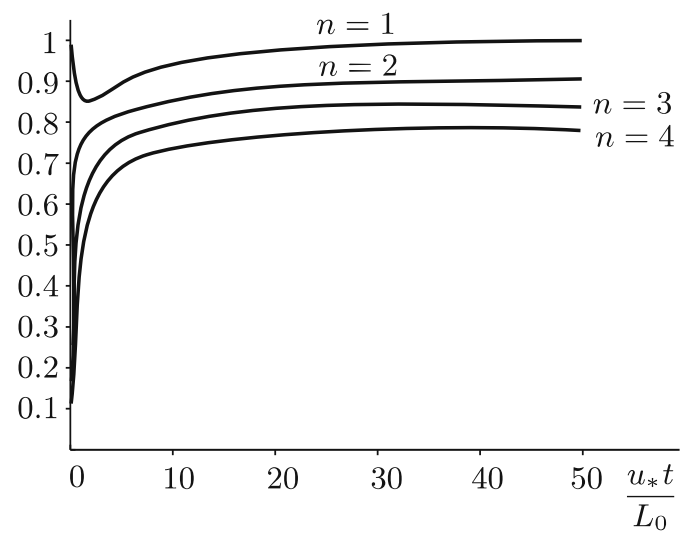

$\mathrm{b}$

Fig. 6. The quotient of $\left(\overline{\overline{x_{2}^{n}}}\right)^{1 / n}$ calculated by numerically simulating the Langevin model for the logarithm layer divided by its value (a) according to the diffusion limit and (b) according to a "corrected" diffusion limit $\kappa_{1} \mapsto 0.85 \kappa_{1}$ : the results correspond to $C_{0}=5.5$ and $b_{1}=0$ and the other parameter values are as in Fig. 1.

We also estimated the skewness and kurtosis from the simulations of the Langevin equation. Their long-time values were respectively found to be 1.6 and 3.4 for the given parameter values. These values should be compared with the respective values 2 and 6 obtained using the diffusion approximation. We note that the long-time values of skewness and kurtosis in the case of the diffusion limit are insensitive to the values of the diffusion constant. They cannot be adjusted by introducing some correction factor. The differences between skewness and kurtosis obtained from the Langevin equation and from the diffusion equation are pure manifestations of the truncation error in the diffusion approximation for a finite value of $C_{0}$. The error is decreased if we increase the value of the Kolmogorov constant.

\section{The value of the Kolmogorov constant}

The conventional approach for analyzing the spread of an admixture in a turbulent flow is to implement the Boussinesq hypothesis leading to the semiempirical equation of turbulent dispersion [2]. The expressions for the diffusion coefficients used in this equation are based on the Reynolds analogy and are fitted to the measurement results. As such, they can be considered calibrated coefficients reflecting observational evidence. Comparing these coefficients with the theoretical diffusivity expressions obtained here yields some interesting insights. It allows determining the value of the Kolmogorov constant $C_{0}$.

The semiempirical equation for wall-normal dispersion in the logarithm layer of wall-induced turbulence contains the diffusion coefficient $s_{t} \kappa u_{*} x_{2}$, where $\kappa u_{*} x_{2}$ follows from the Reynolds analogy with turbulent viscosity [2] and $s_{t}$ is the turbulent Schmidt or Prandtl number, which serves to match model predictions and measurement results. Over the past 50 years and more, many data have been collected for wall-normal admixture dispersion and turbulent heat conduction. They lead to values of $s_{t}$ between 0.9 and 1.1 (see various citations in [2]). Equating the empirical expression $s_{t} \kappa u_{*} x_{2}$ to $\kappa_{1}$ defined by Eq. (46), setting $s_{t}=1$, and setting $\sigma_{22}=1.32 u_{*}^{2}$ in agreement with experimental evidence, we find that $C_{0}=5.5$. A more detailed analysis of measurement data in the logarithm layer and using the Reynolds analogy (turbulent viscosity $=$ turbulent diffusivity) leads to the same result. In general, we can conclude that the value of $C_{0}$ is between 5 and 6 based on the connection between the present theoretical diffusivity expressions and the experimentally determined diffusion coefficients. This compares well with values of $C_{0}$ given in the literature [14]. The values in the literature mostly originate from DNS results for homogeneous forms of turbulence. The 
newness of the present specification of $C_{0}$ is that it is based on results of measuring inhomogeneous wallinduced turbulence. This gives further confidence in the value of the universal Kolmogorov constant to be used in simulating the Langevin equation.

In [2], some indications are also given for the magnitudes and signs of the diffusion coefficients $D_{11}$, $D_{12}$, and $D_{21}$ inferred from behavior in the atmospheric boundary layer. They are in line with the values obtained from Eqs. (41) and (42) if characteristic values are set for $\sigma_{i j}$.

\section{Summary and main conclusions}

The starting point of our investigation was a description of the stochastic process of fluid particle motion in wall-induced turbulence using a Langevin equation. Such an approach agrees with the asymptotic structure of turbulence at a large Reynolds number. We took the white noise term in the equation in accordance with the Lagrangian version of the inertial-sublayer limit of the ordinary Kolmogorov theory of small scales. Some recent measurement results confirm the outcome of the Lagrangian theory of small scales [6]. We did not consider refinements due to intermittency here because intermittency is known to have little effect on the statistical means of particle displacement [9].

The main problem with the Langevin equation is the lack of a unique form for the damping term. This term largely determines the Lagrangian statistics of velocities and displacements. We solved this problem in several steps. First, we took fixed-point Eulerian velocities to be Gaussian. Measurements largely support this assumption. They indicate typical skewness and kurtosis values of Eulerian velocities of 0.3 or less [17][19]. Second, we required well-mixing [16], in other words, the Eulerian interpretation of the Langevin equation should comply with the Gaussian distribution. This requirement leads to conditions on the damping function but is insufficient for a complete specification. This problem is called the nonuniqueness problem [7].

We solved the nonuniqueness problem by requiring a reciprocity analogous to the Onsager reciprocity. Viscous dissipation in the energy equation causes the principle of reciprocity to be applicable only over short times. These times are proportional to the inverse of the Kolmogorov constant $C_{0}$. Reciprocity is therefore only applicable for the leading term in an expansion of the damping function in powers of $C_{0}^{-1}$. Nevertheless, applying the principle seems most rewarding. The uncertainty due to nonuniqueness is deferred to terms with higher powers of $C_{0}^{-1}$. It appears in two parts of the damping function: in the asymmetric part of the linear part of the damping term and in the drift term representing the effect of covariance inhomogeneity. We quantified the magnitude of the asymmetric damping term using recent measurement results for turbulent pipe flow based on particle tracking [29]. We estimated the uncertainty effects in the drift term by analyzing the long-time behavior of the Langevin equation. We thus found that nonuniqueness in both cases has an almost negligible effect on the particle displacement statistics. Their relative effect in both cases seems of the order $O\left(C_{0}^{-2}\right)$ in the stochastic description of particle displacement, which is one order higher than in the Langevin equation for particle velocity! The effect can be disregarded if errors of a few percent are allowed. This leads to a practically unique specification of the Langevin equation for describing the statistics of particle trajectories in wall-induced turbulence at a high Reynolds number. More specifically, the dispersion of a passive admixture can be described by the system of fluctuation equations

$$
\begin{aligned}
\frac{d v_{i}^{\prime}}{d t} & =-\frac{1}{2} C_{0} \varepsilon\left(x_{2}\right) \sigma_{i j}^{-1} v_{j}^{\prime}+\frac{d \sigma_{i 2}}{d x_{2}}+\left(C_{0} \varepsilon\left(x_{2}\right)\right)^{1 / 2} w_{i}(t), \\
\frac{d x_{i}}{d t} & =u_{1}^{0}\left(x_{2}\right) \delta_{i 1}+v_{i}^{\prime},
\end{aligned}
$$

where $\sigma_{i j}=\sigma_{i j}\left(x_{2}\right)=\left\langle u_{i}^{\prime} u_{j}^{\prime}\right\rangle$ is the covariance or Reynolds shear tensor. 
In inhomogeneous turbulence, there is no limit process by which the diffusion approximation becomes exact [32]. To circumvent this difficulty, we introduced the inverse of the Kolmogorov constant as the

small parameter in the long-time approximation of the Langevin model. The resulting diffusion equation involves truncation errors that are reasonably small for the means and standard deviations of displacements but become more serious for higher-order statistics. The diffusion equation appropriate for wall-induced turbulence is given by

$$
\frac{\partial c}{\partial t}+u_{1}^{0}\left(x_{2}\right) \frac{\partial c}{\partial x_{1}}=\frac{\partial}{\partial x_{i}}\left(D_{i j}\left(x_{2}\right) \frac{\partial c}{\partial x_{j}}\right),
$$

where $c$ is the passive admixture concentration and $D_{i j}=D_{i j}\left(x_{2}\right)$ is the turbulent diffusion coefficient defined by Eq. (44). Relating the results in the diffusion limit to the large amount of experimentally established diffusion coefficients over the past 50 years yields a value of the Kolmogorov constant $C_{0}$ between 5 and 6 .

\section{REFERENCES}

1. A. Kolmogoroff, C. R. Acad. Sci., 30, 301-305 (1941).

2. A. S. Monin and A. M. Yaglom, Statistical Fluid Mechanics: Mechanics of Turbulence [in Russian], Vol. 1, Nauka, Moscow (1965); English transl., Dover, Mineola, N. Y. (2007).

3. G. I. Taylor, Proc. London Math. Soc. 2, 20, 196-211 (1922).

4. N. G. van Kampen, Stochastic Processes in Physics and Chemistry (Lect. Notes Math., Vol. 888), North-Holland, Amsterdam (1981).

5. A. S. Monin and A. M. Yaglom, Statistical Fluid Mechanics: Mechanics of Turbulence [in Russian], Vol. 2, Nauka, Moscow (1967); English transl., Dover, Mineola, N. Y. (2007).

6. G. A. Voth, A. La Porta, A. M. Crawford, J. Alexander, and E. Bodenschatz, J. Fluid Mech., 469, 121-160 (2002).

7. J. D. Wilson and B. L. Sawford, Boundary-Layer Meteorology, 78, 191-210 (1996).

8. A. H. Kolmogorov, "Précisions sur la structure locale de la turbulence dans un fluide visqueux aux nombres de Reynolds élevés," in: Mécanique de la turbulence (Colloq. Intern. CNRS, Marseille, Aug.-Sept. 1961), CNRS, Paris (1962), pp. 447-458.

9. M. S. Borgas, Phil. Trans. Roy. Soc. London A, 342, 379-411 (1993).

10. M. S. Borgas and B. L. Sawford, Phys. Fluids, 6, 618-633 (1994).

11. U. Frisch, Turbulence: The Legacy of A. N. Kolmogorov, Cambridge Univ. Press, Cambridge (1995).

12. J. J. H. Brouwers, Phys. Fluids, 19, 101702 (2007).

13. M. S. Borgas and B. L. Sawford, J. Fluid Mech., 279, 69-99 (1994).

14. S. B. Pope, Turbulent Flows, Cambridge Univ. Press, Cambridge (2000).

15. F. Toschi and E. Bodenschatz, "Lagrangian properties of particles in turbulence," in: Annual Review of Fluid Mechanics, Vol. 41, Annual Review, Palo Alto, Calif. (2009), pp. 375-404.

16. D. J. Thomson, J. Fluid Mech., 180, 529-556 (1987).

17. J. Laufer, "The structure of turbulence in fully developed pipe flow," Report No. 1174, http://naca.central. cranfield.ac.uk/reports/1954/naca-report-1174.pdf, NACA (1954).

18. P. S. Klebanoff, "Characteristics of turbulence in a boundary layer with zero pressure gradient," Report No. 1247, http://naca.central.cranfield.ac.uk/reports/1955/naca-report-1247.pdf, NACA (1955).

19. J. F. Morrison, B. J. McKeon, W. Jiang, and A. J. Smits, J. Fluid Mech., 508, 99-131 (2004).

20. J. J. H. Brouwers, Phys. Fluids, 16, 2300-2308 (2004).

21. L. D. Landau and E. M. Lifshits, Statistical Physics: Part 1 [in Russian] (Vol. 5 of Course of Theoretical Physics), Nauka, Moscow (1976); English transl. prev. ed., Pergamon, Oxford (1968).

22. L. E. Reichl, A Modern Course in Statistical Physics, Wiley, New York (1998).

23. O. Bokhove and M. Oliver, Proc. Roy. Soc. London A, 462, 2575-2592 (2006).

24. B. J. McKeon, J. Li, W. Jiang, J. F. Morrison, and A. J. Smits, J. Fluid Mech., 501, 135-147 (2004). 
25. R. Zhao and A. J. Smits, J. Fluid Mech., 576, 457-473 (2007).

26. P. A. Monkewitz, K. A. Chauhan, and H. M. Nagib, Phys. Fluids, 19, 115101 (2007).

27. S. Hoyas and J. Jiménez, Phys. Fluids, 18, 011702 (2006).

28. B. L. Sawford and P. K. Yeung, Phys. Fluids, 12, 2033-2045 (2000).

29. R. J. E. Walpot, C. W. M. van der Geld, and J. G. M. Kuerten, Phys. Fluids, 19, 045102 (2007).

30. B. L. Sawford and P. K. Yeung, Phys. Fluids, 13, 2627-2634 (2001).

31. R. L. Stratonovich, Topics in the Theory of Random Noise, Vol. 1, General Theory of Random Processes, Nonlinear Transformations of Signals and Noise, Gordon and Breach, New York (1967).

32. J. J. H. Brouwers, J. Engrg. Math., 44, 277-295 (2002). 